\title{
Editorial
}

\section{Emergent Techniques and Applications for Big Visual Data}

\author{
Zhijun Fang, ${ }^{1}$ Jenq-Neng Hwang, ${ }^{2}$ Xiaoming Huo, ${ }^{3}$ Hyo-Jong Lee, ${ }^{4}$ and Joachim Denzler ${ }^{5}$ \\ ${ }^{1}$ School of Electronic and Electrical Engineering, Shanghai University of Engineering Science, Shanghai 201620, China \\ ${ }^{2}$ Department of Electrical Engineering, University of Washington, Seattle, WA 98105, USA \\ ${ }^{3}$ Stewart School of Industrial and Systems Engineering, Georgia Institute of Technology, Atlanta, GA 30332, USA \\ ${ }^{4}$ Department of Computer Engineering, Chonbuk National University, Jeonju 54596, Republic of Korea \\ ${ }^{5}$ Department of Mathematics and Computer Sciences, University of Jena, 07743 Jena, Germany \\ Correspondence should be addressed to Zhijun Fang; zjfang@sues.edu.cn
}

Received 20 August 2017; Accepted 20 August 2017; Published 31 October 2017

Copyright (C) 2017 Zhijun Fang et al. This is an open access article distributed under the Creative Commons Attribution License, which permits unrestricted use, distribution, and reproduction in any medium, provided the original work is properly cited.

The exponentially growing number of deployed networked cameras, from fixed to mobile and even airborne, poses strong demands on efficient techniques for better capturing, compressing, networking, computing, and visualization of these huge amounts of visual data. Emerging techniques and adherent applications of big visual data from all kinds of sensors have attracted great attention from industry, academy, and the government. Nonetheless, there are still many challenges that need to be overcome, for example, efficient techniques of analyzing big visual data and general solutions to application related difficulties. Thus, we solicited articles in this special issue that address topics covering key solutions in big visual data: sensing and capturing for big visual data; feature representation and compression of big visual data; networking and dissemination of big visual data; storage and retrieving of big visual data; security and privacy of big visual data; computing and analytics of big visual data; cloud processing and virtualization of big visual data; new applications and services of big visual data; and so forth. Before delving in the particular articles, we first share thoughts with respect to big visual data.

Data capturing and storage are the preliminary processes for big data analyses. While many researchers tried to define what big data are, the more popular description for big data arises from the properties they possess. For example, the three defining dimensions for big data are volume, variety, and velocity, which are called the Three Vs. When capturing data, the above properties should be considered in order to provide high quality data. However, digitizing and labeling a massive volume of data are time-consuming and sometimes it goes against personal privacy protection. Therefore, massive discussions and research concerning security and privacy of big visual data must be addressed as well.

A great deal of techniques are available to extract structure information from large-scale data. It is worth mentioning that machine learning is a powerful tool for identification/prediction of unknown test data by learning models from either the past history or available training data. In recent years, deep learning using convolutional neural networks (CNNs) plays a crucial role in feature representation, system modeling, and class prediction by learning from a huge amount of training data. However, it always requires expensive computations and the model has extremely high complexity which limits its universality. Recently, researchers focus on methods of reducing the computational complexity of deep learning and improving the scalability of deep learning. A typical application is face detection from largescale real-world visual data.

Analyzing big data is in the end to improve the service for the society. New emerging applications are varying from global climate change prediction to daily life applications. For example, health care, customer behavior, intelligent transportation, recommendation system, and energy distribution. Other applications are, for example, exploring and understanding social dynamics from social media data and urban computing based on taxi trajectories to explore knowledge about a city and its citizens. In this special issue, several applications related to big visual data are investigated. 
The editorial committee of this special issue received 20 submissions of which 5 papers were accepted for publication, giving an acceptance rate of $25 \%$. Various applications and techniques related to big visual data are discussed. More specifically, Y. Yu et al. proposed a physics based 3D character animation method based on the open dynamics engine (ODE) to avoid the big data acquisition from motion capturing equipment. X. Sun et al. proposed a gradient domain image filtering-based low-light image enhancement method, which effectively extracts the illumination component of the underlying image. To efficiently deal with the problem of information overload for online users, F. Zhang et al. proposed a novel preferential diffusion movie recommendation algorithm considering the significance of the target user's nearest neighbors. Large amounts of medical CT images have been collected to establish a big medical data library. An active shape model is applied by X. Duan et al. to automatically reconstruct aortic dissection to assist operations of doctors.

Moreover, to handle large-scale text automatic processing, which uses an event network to conceal lexical relations in events and logical relations between events in document, Y. Shi et al. took advantage of the expanded Chinese Event Corpus (CEC) as data source and prior knowledge of manifestation rules of event and relation as the guide, to establish a knowledge-based rule of event manifestation, so as to achieve automatic building and improve text processing performance of event network.

As a hot research topic, more and more conferences and journals call for articles on big visual data analysis, which enhances interdisciplinary collaborations and creates new $\mathrm{R} \& \mathrm{D}$ domains. We believe that more and more theoretical advances and killer applications of big visual data will be available in the next years.

\author{
Zhijun Fang \\ Jenq-Neng Hwang \\ Xiaoming Huo \\ Hyo-Jong Lee \\ Joachim Denzler
}




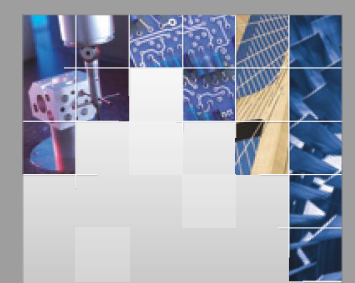

\section{Enfincering}
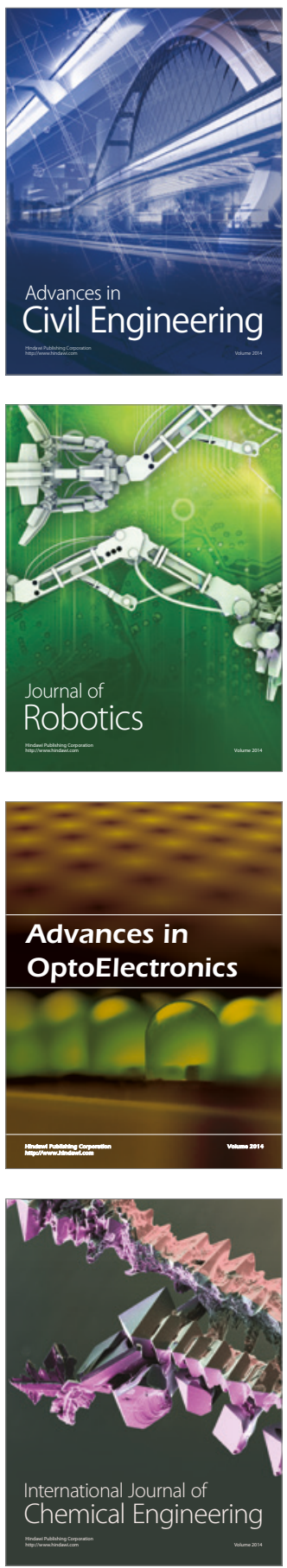

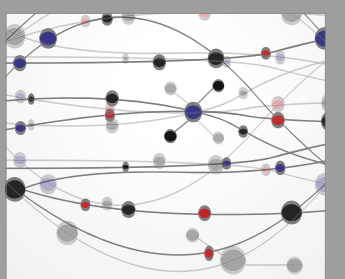

The Scientific World Journal

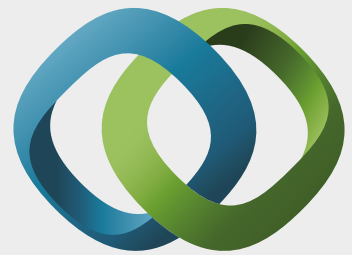

\section{Hindawi}

Submit your manuscripts at

https://www.hindawi.com
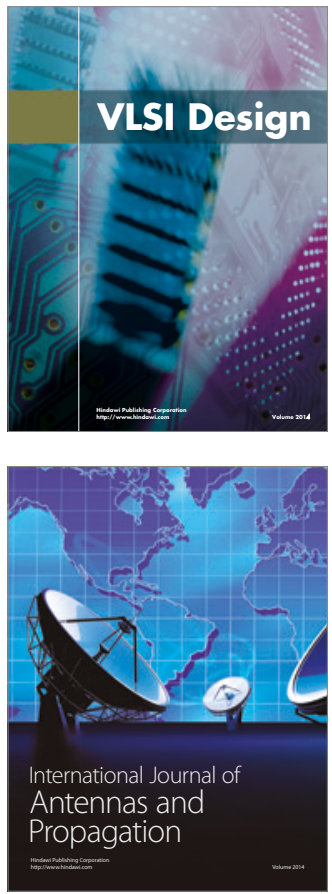

\section{Rotating}

Machinery
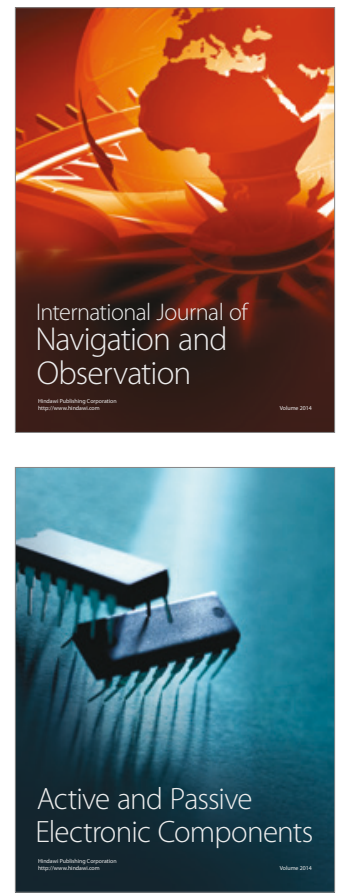
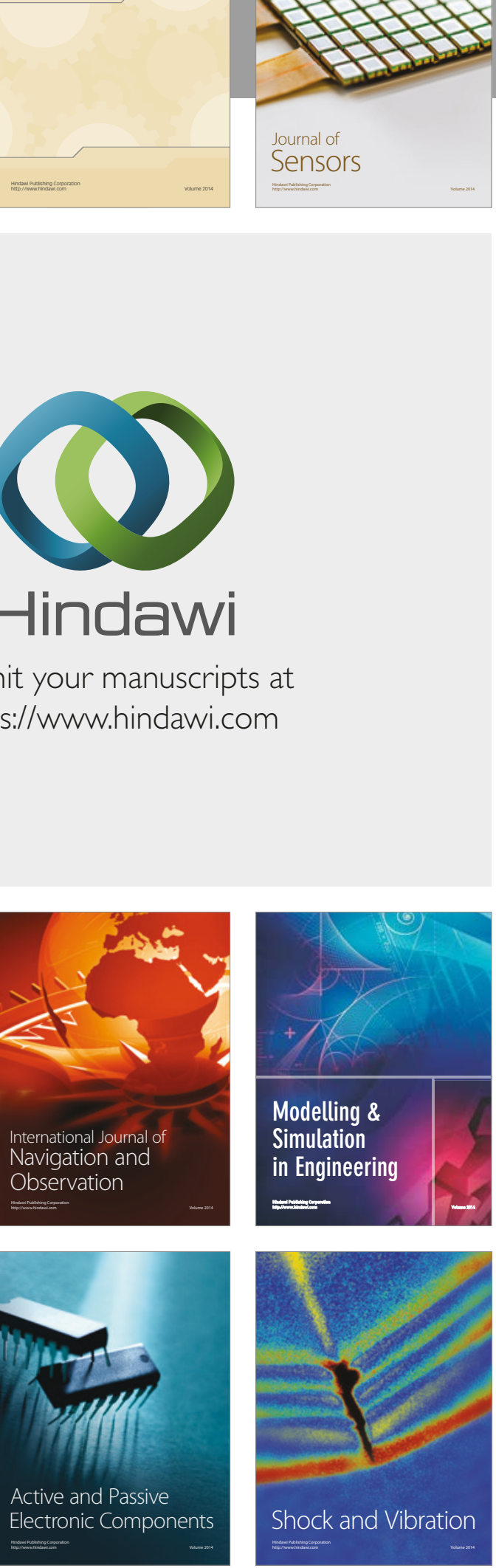
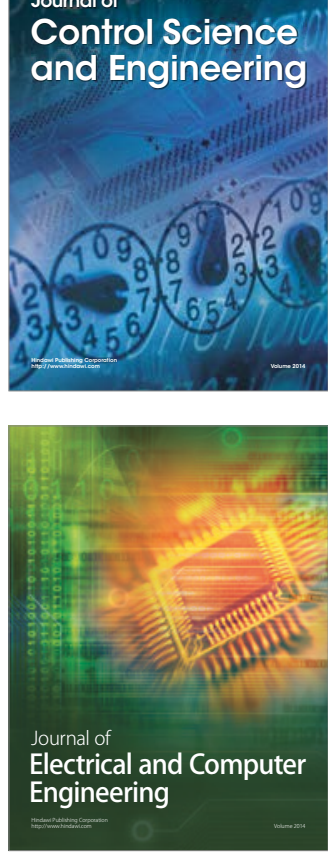

Distributed

Journal of

Control Science

and Engineering
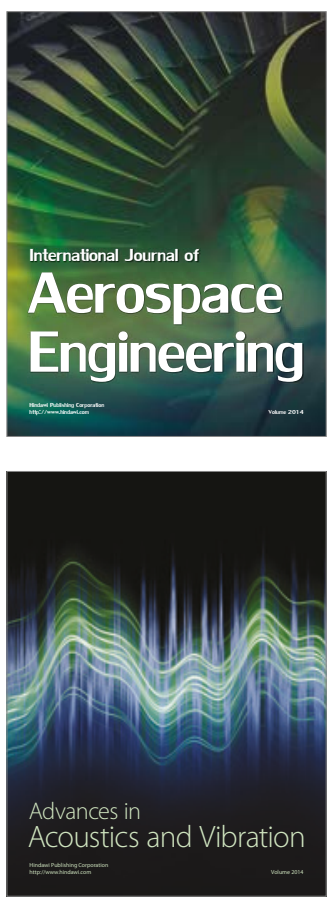

Sensor Networks 